Research Article

\title{
Two-Dimensional Numerical Study on the Migration of Particle in a Serpentine Channel
}

\author{
Yi Liu, Qucheng Li, and Deming Nie \\ Institute of Fluid Mechanics, China Jiliang University, Hangzhou, China \\ Correspondence should be addressed to Deming Nie; nieinhz@gmail.com
}

Received 2 February 2018; Accepted 4 April 2018; Published 17 May 2018

Academic Editor: Martin Seipenbusch

Copyright ( 92018 Yi Liu et al. This is an open access article distributed under the Creative Commons Attribution License, which permits unrestricted use, distribution, and reproduction in any medium, provided the original work is properly cited.

In this work, the momentum exchange scheme-based lattice Boltzmann method is adopted to numerically study the migration of a circular particle in a serpentine channel for the range of $20 \leq \mathrm{Re} \leq 120$. The effects of the Reynolds number, particle density, and the initial particle position are taken into account. Numerical results include the streamlines, particle trajectories, and final equilibrium positions. Close attention is also paid to the time it takes for the particle to travel in the channel. It has been found that the particle is likely to migrate to a similar equilibrium position irrespective of its initial position when Re is large. Furthermore, there exists a critical solid-to-fluid density ratio for which the particle travels fastest in the channel.

\section{Introduction}

Solid particles immersed in a viscous fluid lead to a two-phase flow problem, which is very common in nature and in many industrial processes, including atmospheric currents, aerosol deposition, fluidized beds, and so on. The motion and dynamics of particles suspended in a fluid is fundamental to understanding suspension hydrodynamics. Over the past decade, great progress has been made for microfluidic devices because of several benefits over conventionally sized systems, such as small volume of sample and reagent, low energy consumption, high efficiency, and enhanced analytical sensitivity. In microfluidic devices, manipulation and separation of particles are usually necessary in the processes of enzymatic analysis, DNA analysis, and sample separation. However, it is necessary to focus the samples in a tight stream before separation, sorting, or analysis in order to ensure that these samples are passing through the microchannels quickly. Therefore, understanding the behavior and characteristics of particle suspensions in microfluidics is helpful to provide insight into the design of microfluidic channels [1].

There are several methods of particle focusing that have been developed and used in microfluidic systems. Inertial focusing is usually adopted to align the particles along a tight stream, which has the advantage over other methods because it does not require external forces or multiple streams to focus particles. The most famous phenomena of inertial focusing may be the Segré-Silberberg effect [2], which is a fluid dynamic separation effect where a dilute suspension of neutrally buoyant particles flowing in a tube equilibrates at a distance $\sim 0.6 R$ (tube radius) from the tube center. Later a full analytical solution of the forces that dominate particles in Poiseuille flow was provided by Ho and Leal [3]. They [3] showed that particles migrate from the center of a channel towards the wall due to shear-induced lift forces and are rejected from the channel perimeter by wall-induced lift forces creating a stable equilibrium at a distance of $0.6 R$ from the center of the channel. Feng et al. [4] were the first to observe the Segré-Silberberg effect for the motion of a single circular particle in plane Poiseuille flow by using the finiteelement method. Pan and Glowinski [5] simulated the motion of multiple circular particles in plane Poiseuille flow. Their results [5] showed that the collisions between particles have a significant influence on the inertial migration of particles. Chun and Ladd [6] investigated the inertial migration of neutrally buoyant particles in a square duct. For the case of elliptical particles, Yi et al. [7] reported that the particles fluctuate about an averaged position at low Reynolds numbers while they converge to an equilibrium position on each side of the channel center at high Reynolds 
numbers. Chen et al. [8] studied the motion of two spherical particles in tube flow through the lattice Boltzmann method (LBM). They reported an oscillatory state of motion for two spherical particles with different radii in opposite sides. Similarly, Abbas et al. [9] simulated the motion of a spherical particle in a square channel flow. They demonstrated that there exist two states for the migration of particle which are cross-streamline stage and cross-lateral stage, respectively. In addition, they [9] showed that the former stage is much faster than the latter one. Recently, Jiang et al. [10] investigated the migration of particles in a symmetrical serpentine channel through a three-dimensional LBM. They focused on the influence of the Dean flow on particle focusing in a serpentine channel. Their results showed that the alternation of the Dean flow direction has special hydrodynamic effects to focus or separate particles of different sizes as the flow intensity becomes stronger.

So far, the inertial focusing has numerous applications in microparticle manipulation ranging from microfluidic cell sorting to particle separation and ordering [11-13]. However, due to the compact size of microfluidic devices, the majority of the biomedical processes of inertial focusing are carried out in the curved channels, such as expansioncontraction array channel, spiral channel, and serpentine channel. In comparison with the straight channel, the flow conditions are very different in the curved channels. For instance, it is naturally expected that the boundary layer separation will take place in the corners of a serpentine channel, which usually affects the flow structure and of course the motion of particles. The centrifugal force in the curved channels is also a key factor which may dominate the migration of particles and should be taken into account [14]. Furthermore, it is possible that the hydrodynamic interactions between particles in the curved channels are more complex which are usually responsible for the aggregation of particles in the finite-Reynolds-number regime. Unlike the straight channel, it is very difficult to derive a detailed mathematical description of the forces that dominate particles in the curved channels due to the complex nature of flow. At present, much of the development in the curved channels has followed an empirical approach which usually fails to predict the equilibrium position of particles. Therefore, a more complete understanding of the migration of particles in these channels is needed to provide help with the design of microfluidic channels and to further enhance the focusing of particles. However, attempts to study the flow characteristics as well as the motion of particles in a curved channel are rarely reported in the past, most of which are involving experimental work. Little effort has been paid to the study of the migration of particles in a curved channel from a numerical aspect. This motivates the present work.

Among all the types of curved channel, the serpentine channel with linear structure is an optimal choice due to its small footprint and easy parallelization. Furthermore, experimental work has shown that the serpentine channel can achieve focusing and separation within a much shorter length due to the assistance of secondary flow [15]. The similar behavior was also demonstrated in our recent work [16], which presents some primitive results involving particle migration in a serpentine channel. On basis of this, we aim to further present a thorough two-dimensional numerical study of a single particle migrating in a serpentine channel through direct numerical simulations (DNS). The effects of the Reynolds number as well as the initial position of particle on the particle migration are studied. Numerical results include the streamline, particle trajectory, and the equilibrium position of particle. Close attention will be paid to the time it takes for the particle to travel in the serpentine channel. We hope the simulation results would be helpful for the designing of microfluidic devices.

\section{Numerical Model}

2.1. Lattice Boltzmann Method. In this work, the lattice Bhatnagar-Gross-Krook (LBGK) Boltzmann method proposed is used to solve the fluid flow [17]:

$$
f_{i}\left(\mathbf{x}+\mathbf{c}_{i} \Delta t, t+\Delta t\right)-f_{i}(\mathbf{x}, t)=-\frac{1}{\tau}\left[f_{i}(\mathbf{x}, t)-f_{i}^{(\mathrm{eq})}(\mathbf{x}, t)\right],
$$

where $f_{i}(\mathbf{x}, t)$ and $f_{i}^{(\mathrm{eq})}(\mathbf{x}, t)$ are the distribution functions and corresponding equilibrium distribution functions associated with the $i$ th discrete velocity direction $\mathrm{c}_{i} . \Delta t$ is the time step and $\tau$ is the relaxation time, respectively. In the two-dimensional nine-velocity lattice (D2Q9), the model proposed by Qian et al. [17] is adopted here,

$$
\mathbf{c}=c\left[\begin{array}{ccccccccc}
0 & 1 & 0 & -1 & 0 & 1 & -1 & -1 & 1 \\
0 & 0 & 1 & 0 & -1 & 1 & 1 & -1 & -1
\end{array}\right] .
$$

The lattice speed is $c=\Delta x / \Delta t$, where $\Delta x$ is the lattice spacing. $f_{i}^{\text {(eq) }}(\mathbf{x}, t)$ for this lattice is

$$
f_{i}^{(\mathrm{eq})}=w_{i} \rho_{\mathrm{f}}\left[1+\frac{\mathbf{c}_{i} \cdot \mathbf{u}}{c_{\mathrm{s}}^{2}}+\frac{\left(\mathbf{c}_{i} \cdot \mathbf{u}\right)^{2}}{2 c_{\mathrm{s}}^{4}}-\frac{\mathbf{u}^{2}}{2 c_{\mathrm{s}}^{2}}\right],
$$

where $\rho_{\mathrm{f}}$ is the fluid density, $c_{\mathrm{s}}$ is the sound speed, and $w_{i}$ is the weight coefficient given by

$$
\begin{aligned}
& w_{i}=\left\{\begin{array}{cc}
4 / 9, & c_{i}^{2}=0 \\
1 / 9, & c_{i}^{2}=c^{2} \\
1 / 36, & c_{i}^{2}=2 c^{2}
\end{array}\right\}, \\
& c_{\mathrm{s}}=\frac{c}{\sqrt{3}} .
\end{aligned}
$$

The fluid density $\rho_{\mathrm{f}}$ and velocity $\mathbf{u}$ can be calculated by the following formula:

$$
\begin{gathered}
\rho_{\mathrm{f}}=\sum_{i} f_{i}, \\
\rho_{\mathrm{f}} \mathbf{u}=\sum_{i} c_{i} f_{i} .
\end{gathered}
$$

The Navier-Stokes equations can be obtained from the lattice Boltzmann equation (LBE) through a ChapmanEnskog expansion proposed by He and Luo [18]. 


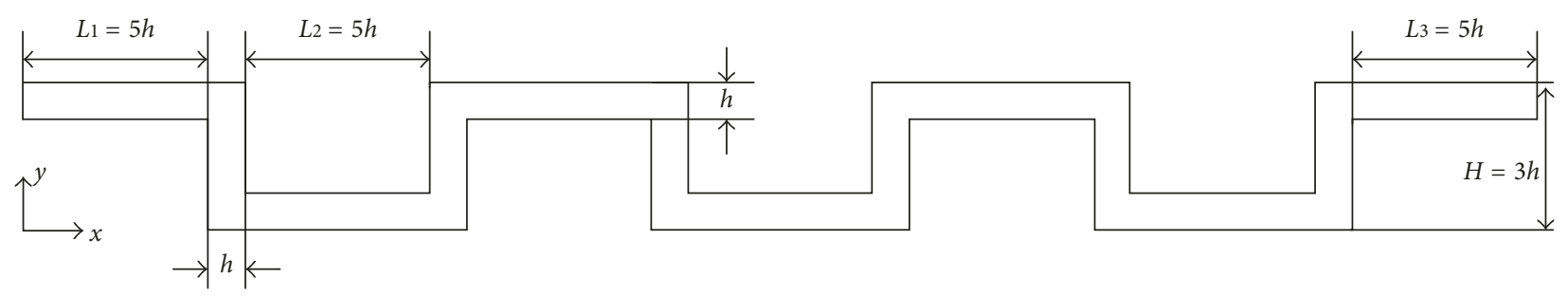

FIgure 1: Physical model of the present work.

2.2. Problem Description. In this work, we aim to numerically study the migration of a circular particle in a serpentine channel. The physical model is shown in Figure 1. The width of the channel is denoted as $h$. Other parameters such as $L_{1}$, $L_{2}, L_{3}$, and $H$ are shown in Figure 1, which are set to be $L_{1}=L_{2}=L_{3}=5 h$ and $H=3 h$. The diameter and density of the particle are expressed by $d$ and $\rho_{\mathrm{p}}$, respectively. At the inlet, a parabolic flow with the maximum velocity of $U_{0}$ is applied, while the fully developed condition is applied at the outlet. No slip boundary is used on all the channel walls. In the simulations, the parameters are chosen as follows: $\rho_{f}=1, d=$ $10, h=8 d$, and $\mathrm{U}_{0}=0.05$ (in lattice unit).

The channel Reynolds number Re in this work is defined as

$$
\operatorname{Re}=\frac{U_{0} h}{v} .
$$

In order to avoid the influence of the inlet on the particle motion, the particle is always placed $20 \mathrm{~d}$ from the inlet, which is released after the flow is fully developed. In this work, we focus on the effect of the Reynolds number, the particle initial position, as well as the density of particle on the final equilibrium position of particle, and its migration trajectory.

\section{Validation}

In order to validate the computational model in this work, the migration of a circular particle in a straight channel is numerically tested. We compare the present results with the previous results proposed by Chen et al. [8], as shown in Figure 2. The parameters used here are the same as those in [7]. Explicitly, the diameter of the circular particle is $d=22$ and the width of the straight channel is $H=200$, which leads to the size ratio $K=d / H=0.11$. The equilibrium position of the particle $Y_{\text {eq }}$, which is the vertical position of the particle to the width of the channel, at different Reynolds numbers $(\operatorname{Re}=20,40,60,80,100,120,160,180$, and 200) is shown in Figure 2. The Segré-Silberberg effect is realized, with the equilibrium position a little outside the midpoint between the wall and the channel centerline. In addition, the final equilibrium position of particle is closer to the channel centerline when increasing the Reynolds number.

In Table 1, we present the relative errors between our results and those in [11] at different Reynolds numbers, which shows a good agreement.

\section{Results and Discussion}

4.1. Steady Flow Field. In the simulations, the particle is released after the flow field is fully developed. To better

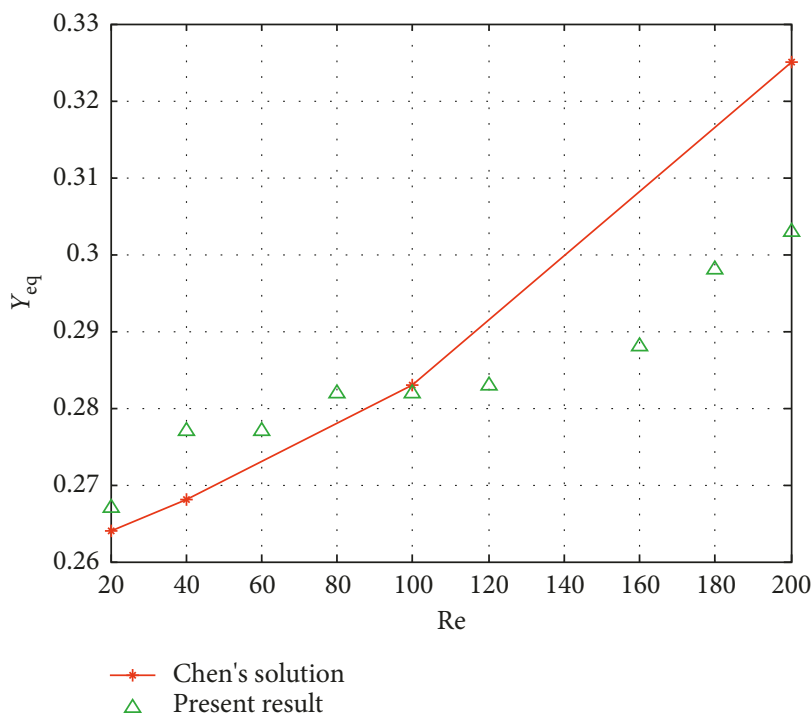

FIgURE 2: Comparison of the equilibrium position of a particle migrating in a straight channel.

TABLE 1: Relative errors of different Reynolds numbers.

\begin{tabular}{ll}
\hline $\mathrm{Re}$ & $(\%)$ \\
\hline 20 & 1.14 \\
40 & 3.36 \\
100 & 0.35 \\
200 & 6.77 \\
\hline
\end{tabular}

illustrate the flow field in the serpentine channel, we present the steady streamlines at different Reynolds numbers $(\operatorname{Re}=20,70$, and 120$)$ in Figure 3. Only the local enlargement of steady flow field is shown because of large computational domain.

It is hard to observe the recirculation zones when the Reynolds number is small, such as $\operatorname{Re}=20$, as shown in Figure 3(a). As a result, the distortion of the streamlines is not significant. However, the recirculation zones are becoming more notable for larger Re, as one can see in Figures 3(b) and 3(c). Totally speaking, there are two types of corners in the present serpentine channel: corners with inwards right angle and those with outwards right angle, which lead to two types of recirculation zone. It is observed that there always exists a recirculation zone for each corner when Re is large. Furthermore, the rotation of recirculation zones on the upper wall is always counterclockwise while the opposite is true for 


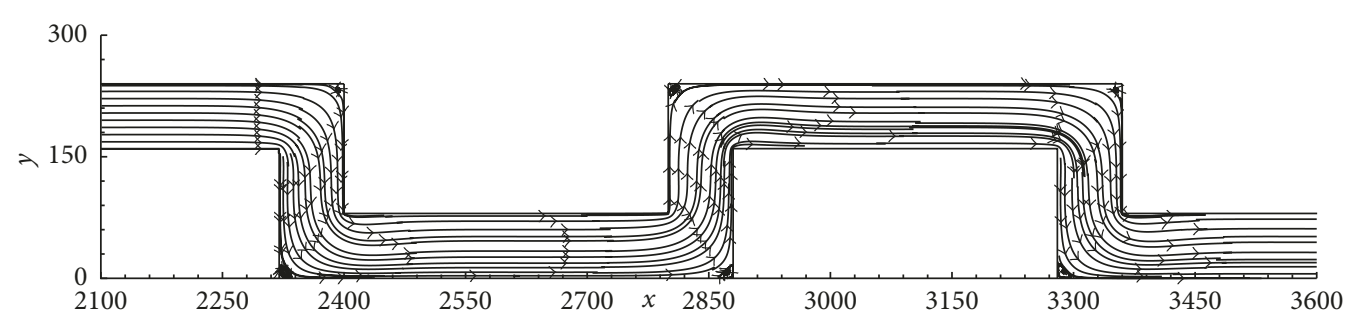

(a)

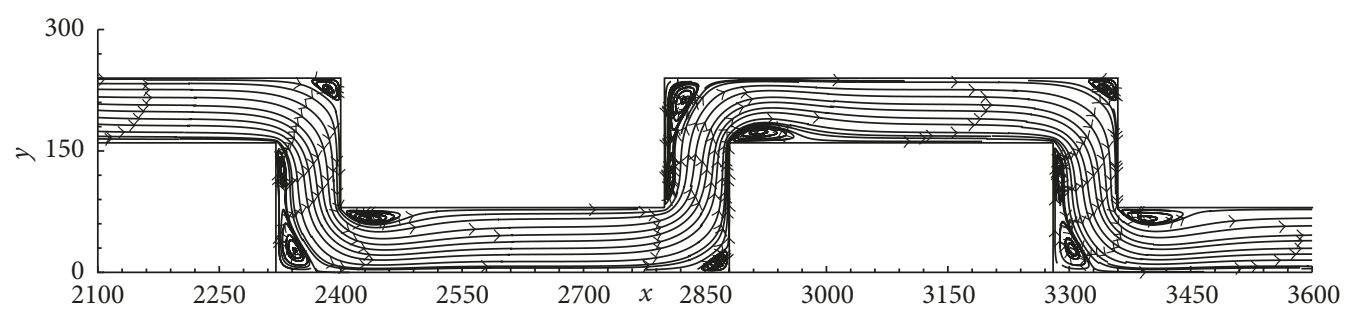

(b)

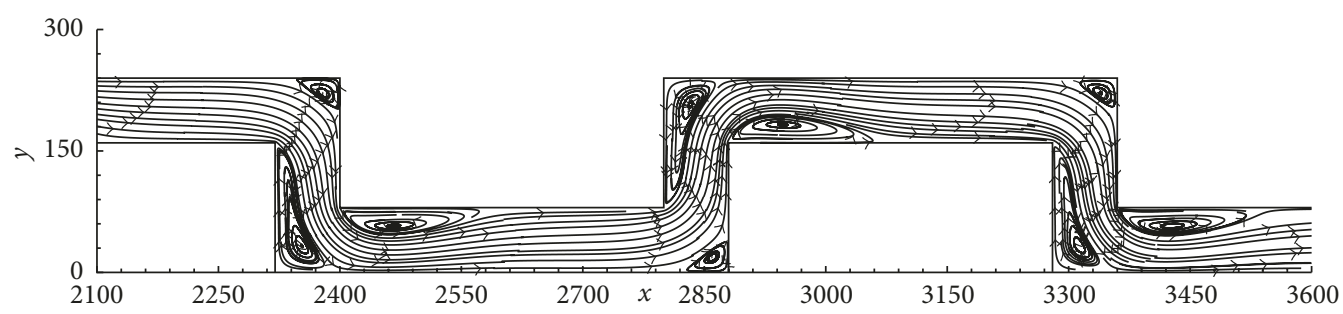

(c)

Figure 3: Streamlines of steady flow field at (a) $\operatorname{Re}=20$, (b) $\operatorname{Re}=70$, and (c) $\operatorname{Re}=120$.

those on the bottom wall. Due to the recirculation zones, the distortion of the streamlines becomes significant when the Reynolds number is large, such as $\operatorname{Re}=120$, which is expected to considerately influence the migration of particle in the channel.

4.2. The Effect of the Reynolds Number. In this section, we study the effect of the Reynolds number on the particle migration in the serpentine channel shown in Figure 1. The density of the particle is fixed at $\rho_{\mathrm{p}}=1.0$, suggesting that the particle is neutrally suspended. Here, a parameter $q$ is introduced to describe the initial lateral position of the particle in the channel which is defined by the distance of particle to the upper channel wall normalized by the channel width $h$. As a result, the value of $q=0.5$ indicates that the particle is initially placed on the channel centerline. In what follows, we focus on the particle trajectory as well as the final equilibrium position of the particle under different channel Reynolds numbers.

Figure 4 shows the particle trajectories at $\mathrm{Re}=20,70$, and 120 for different initial particle positions. As one can see, the effect of $\mathrm{Re}$ on the particle migration in the channel is significant. To some extent, the particle trajectories are similar to the streamlines shown in Figure 3. In comparison with the result of $\operatorname{Re}=20$, the particle is driven farther away from the channel wall after passing through each bend when increasing $\mathrm{Re}$, which is more significant for small values of $q$, as shown in Figure 4. This is due to the fact that the larger the Reynolds number, the larger the centrifugal force experienced by the particle.

The effect of $q$ on the particle trajectories is shown in Figure 5. The particle trajectories of different $q$ are almost parallel to each other for each Reynolds number. It is observed that the dependence of particle migration on the value of $q$ is stronger when Re is smaller. In other words, the final equilibrium position of particle is more sensitive to the value of $q$ for smaller Reynolds number. For instance, no visible difference is observed for all results except that of $q=0.5$ when $\operatorname{Re}=120$, the largest Reynolds number studied in the work, as one can see in Figure 5(c). In order to provide a better understanding, we summarize the dependence of the final equilibrium positions of particle on Re as well as $q$ in Table 2, which further demonstrates that the effect of $q$ on the particle migration is more significant at smaller Re. It is also clear that the particle eventually stays on the channel centerline for the range of Re studied when $q=0.5$.

High efficiency is occasionally required in the microfluidic devices such as fast separation. In these cases, the particles are expected to travel fast in the serpentine channel. As a result, much attention should be paid to the time needed for the particles to travel in the channel. We carried out a preliminary study upon this issue. The dependence of $T^{*}$ on the value of $q$ is shown in Figure 6 for different Re. It should be stated that $T^{*}$ is normalized by $T_{0}$ which is determined through $T_{0}=d / U_{0}$. As shown in Figure 6, all the 


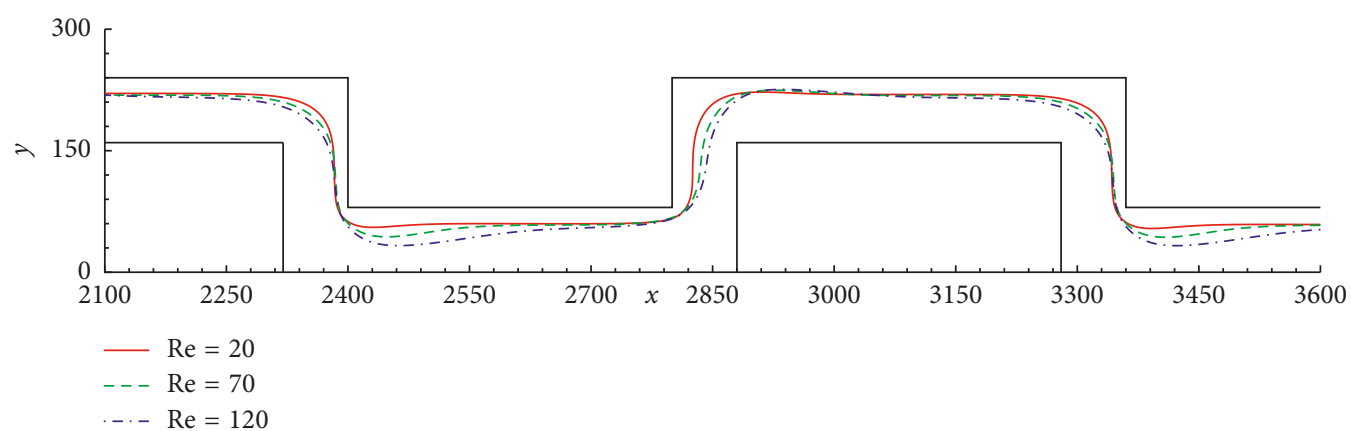

(a)

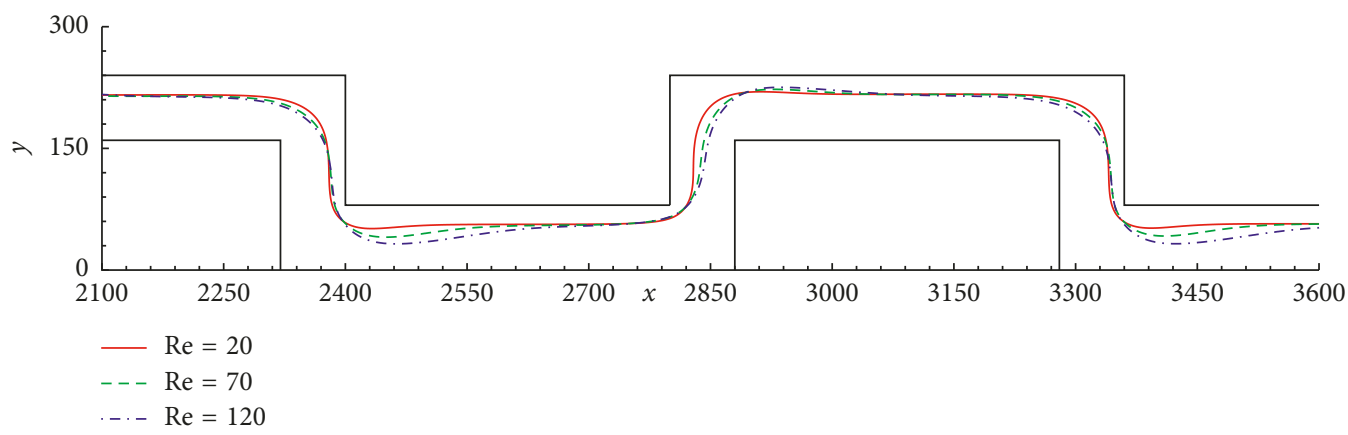

(b)

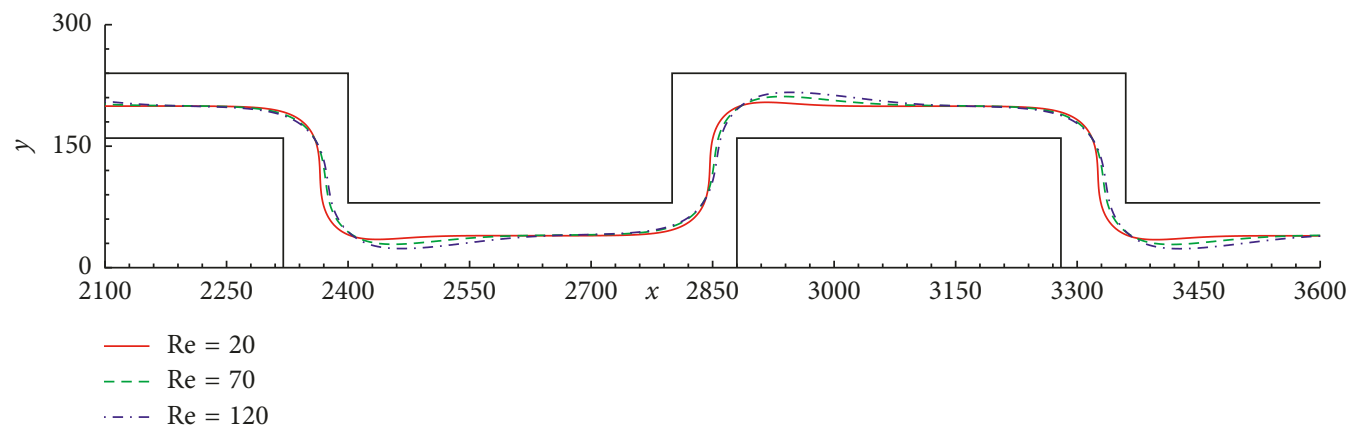

(c)

Figure 4: Particle trajectories at $\operatorname{Re}=20,70$, and 120 for different $q$ : (a) $q=0.1$, (b) $q=0.3$, and (c) $q=0.5$.

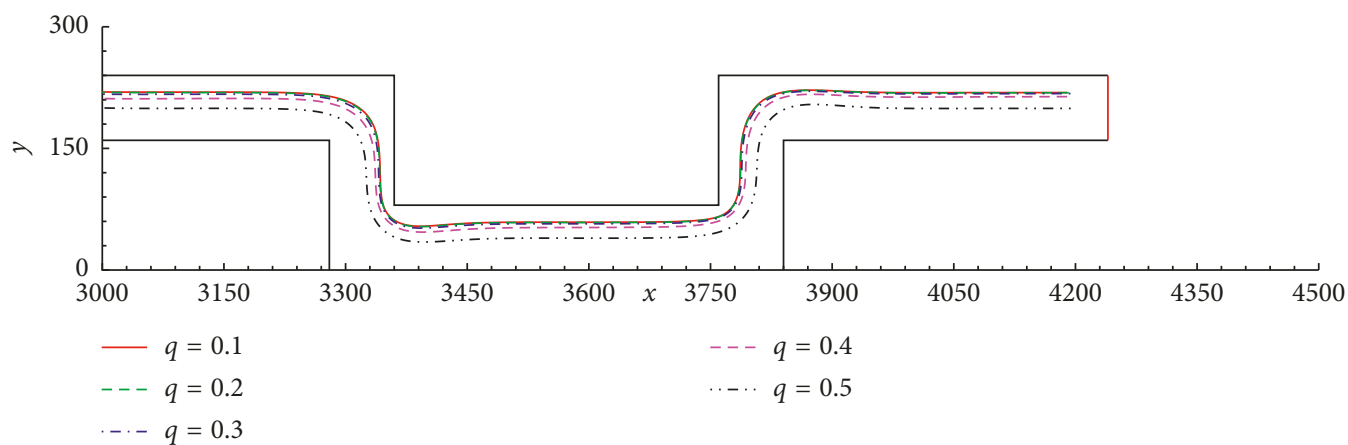

(a)

FiguRe 5: Continued. 


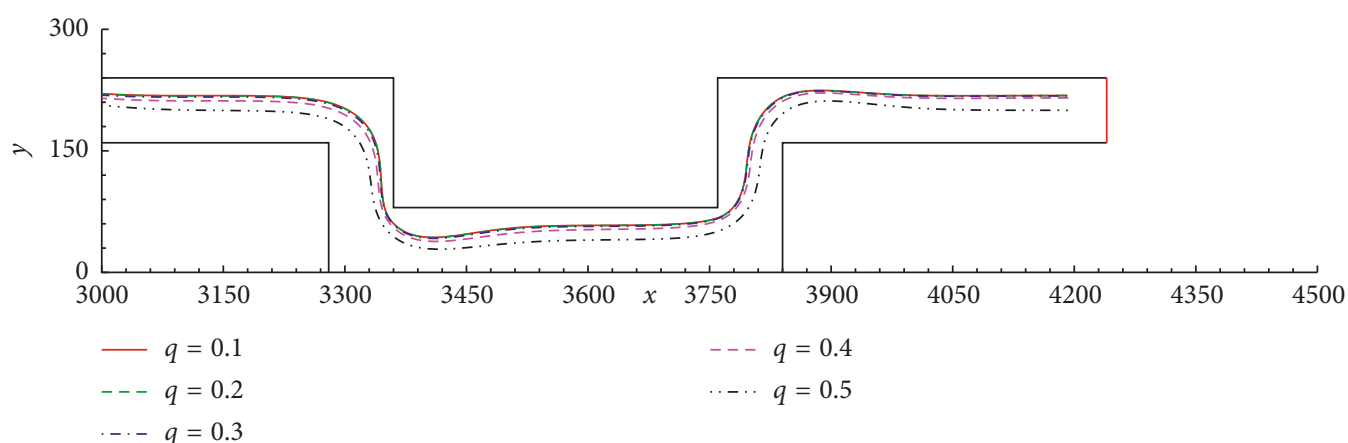

(b)

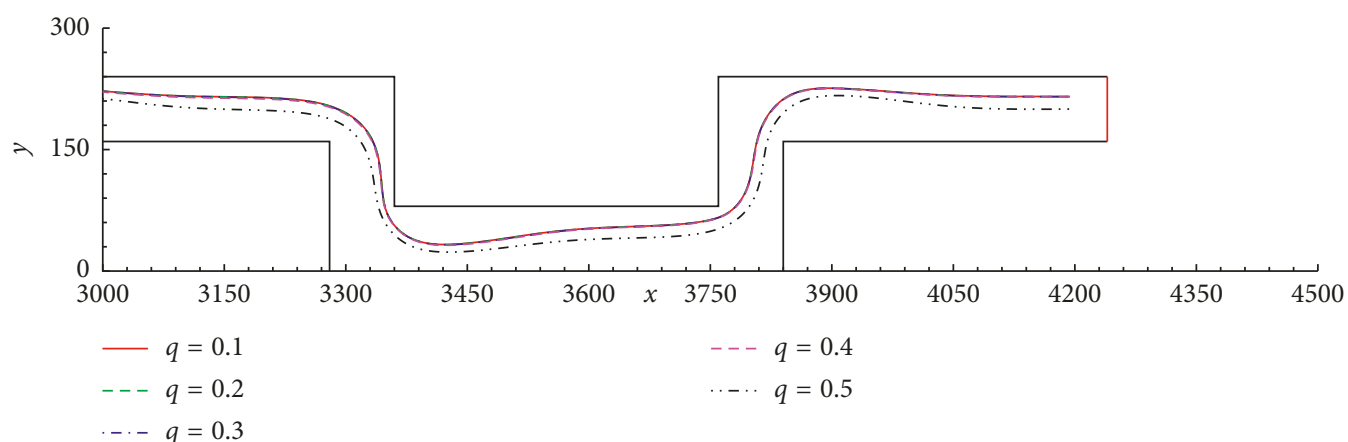

(c)

Figure 5: The effect of $q$ on particle trajectories: (a) $\operatorname{Re}=20$, (b) $\operatorname{Re}=70$, and (c) $\operatorname{Re}=120$.

TABLE 2: Dependence of the final equilibrium position on Re as well as $q$.

\begin{tabular}{lccc}
\hline $\operatorname{Re}$ & 20 & 70 & 120 \\
\hline$q=0.1$ & 0.265 & 0.271 & 0.305 \\
$q=0.2$ & 0.268 & 0.273 & 0.308 \\
$q=0.3$ & 0.280 & 0.278 & 0.310 \\
$q=0.4$ & 0.328 & 0.308 & 0.314 \\
$q=0.5$ & 0.509 & 0.500 & 0.500 \\
\hline
\end{tabular}

results decrease as $q$ increases when $q<0.5$, while the opposite is true when $q>0.5$. This leads to the fact that the time $T^{*}$ reaches its minimum value when $q=0.5$ for all cases, suggesting that the particle will travel fastest if it is initially placed on the channel centerline. In addition, the results are more likely to be symmetrical when Re is smaller, such as $\mathrm{Re}=20$, which is due to the symmetrical flow conditions. However, the recirculation zones resulting from corners become significant as increasing Re, which destroys the flow symmetry. Furthermore, it is found that the time needed for the particle to travel in the channel has a quadratic-like relationship with the value of $q$ for small $\operatorname{Re}(\operatorname{Re}=20)$ :

$$
T^{*}=1632 q^{2}-1629 q+859 \text {. }
$$

To further study the migration behavior of the particle in the serpentine channel, we also pay close attention to the evolution of particle orientation $\theta$ which has an initial value of $\pi / 2$, as shown in Figures 7 and 8 , which present the corresponding results of $q=0.1-0.3$ and $q=0.5$ at different Reynolds numbers, respectively. As we can observe in

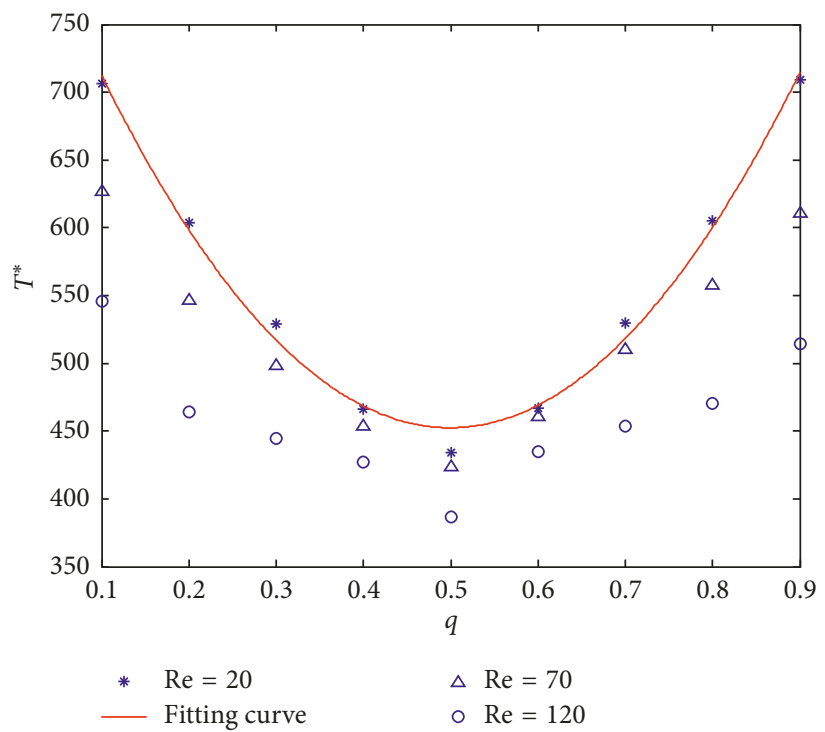

Figure 6: Time needed for the particle to travel in the channel for different values of $q$.

Figure 7, the particle always rotates counterclockwise when traveling in the channel if $q<0.5$ irrespective of Re. In addition, the smaller the value of $q$ is, the faster the particle rotates. This is because the particle experiences larger gradient of fluid velocity if it is closer to the channel wall.

Figure 8 shows a different pattern of particle motion in the serpentine channel when $q=0.5$. Instead of rotating counterclockwise, the particle will oscillate if it is initially 


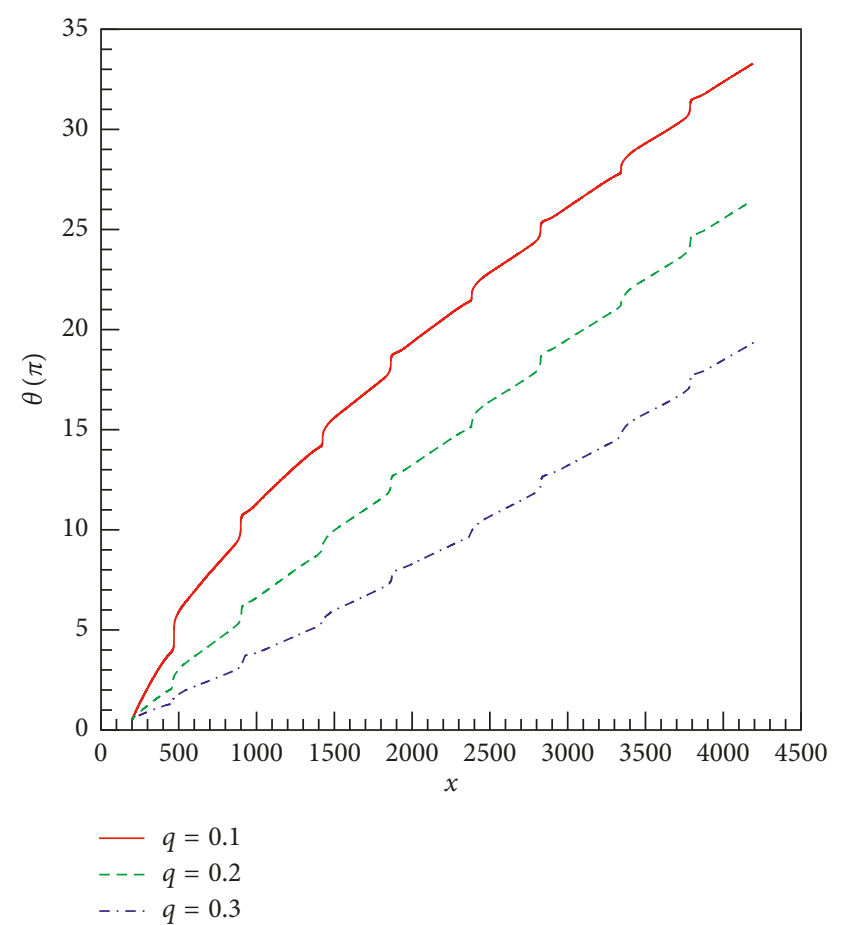

(a)

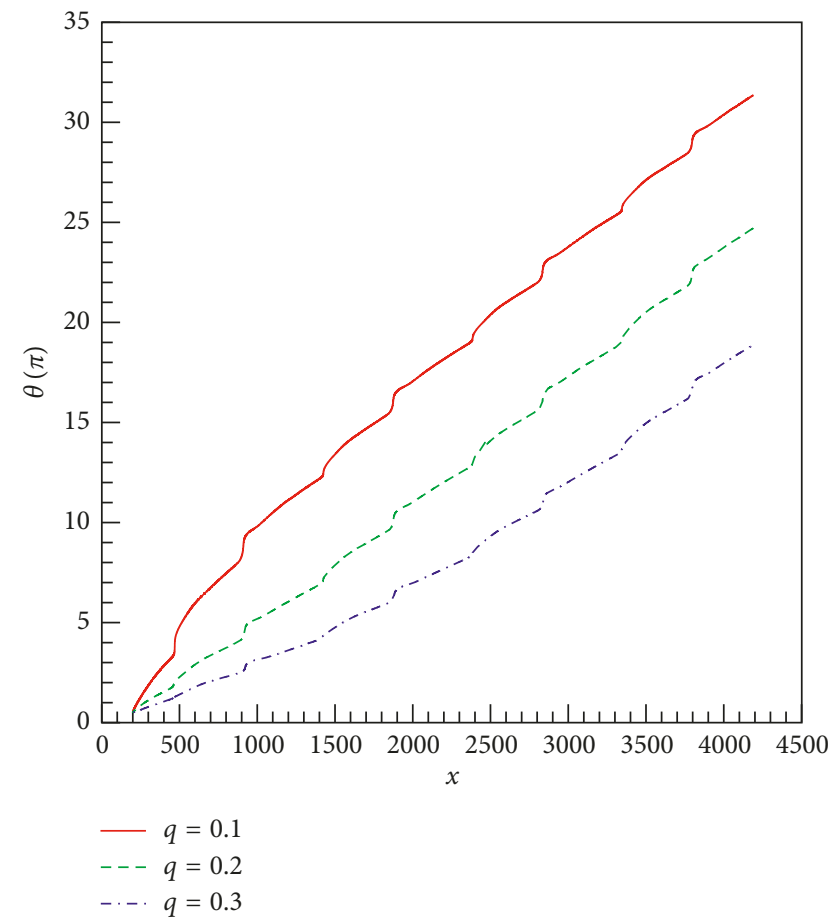

(b)

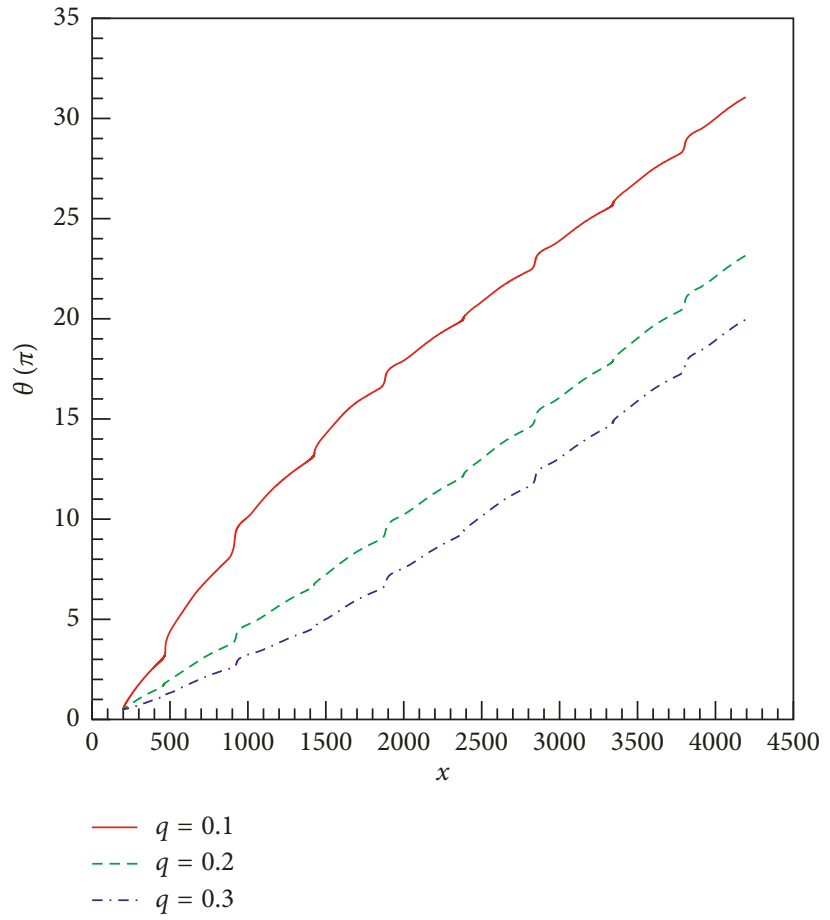

(c)

Figure 7: The orientation of the particle for $q=0.1,0.2$, and0.3 at different Reynolds numbers: (a) $\operatorname{Re}=20,(\mathrm{~b}) \operatorname{Re}=70$, and (c) $\operatorname{Re}=120$ (also appeared in [16]).

placed on the channel centerline for the range of Re in this work. Especially, it is observed that the particle is oscillating around $\theta=\pi / 2$ when traveling in the channel for large Reynolds numbers, such as $\operatorname{Re}=70$ and 120 , as shown in Figure 8.
4.3. The Effect of Particle Density. The particle inertia is central to the behavior of the particle suspended in fluids because it determines how the velocity of the particle decays due to fluid drag. As is known to all, the particle inertia strongly depends on its density. Consequently, we focus on 


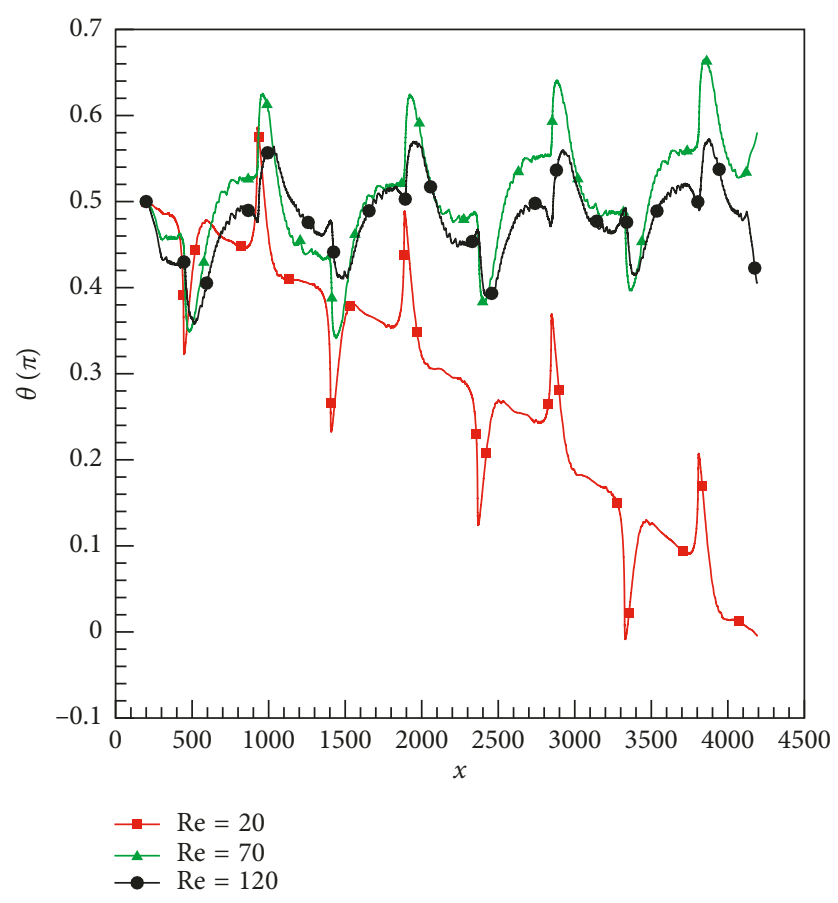

FIGURE 8: The orientation of the particle for $q=0.5$ at different Reynolds numbers (also appeared in [16]).

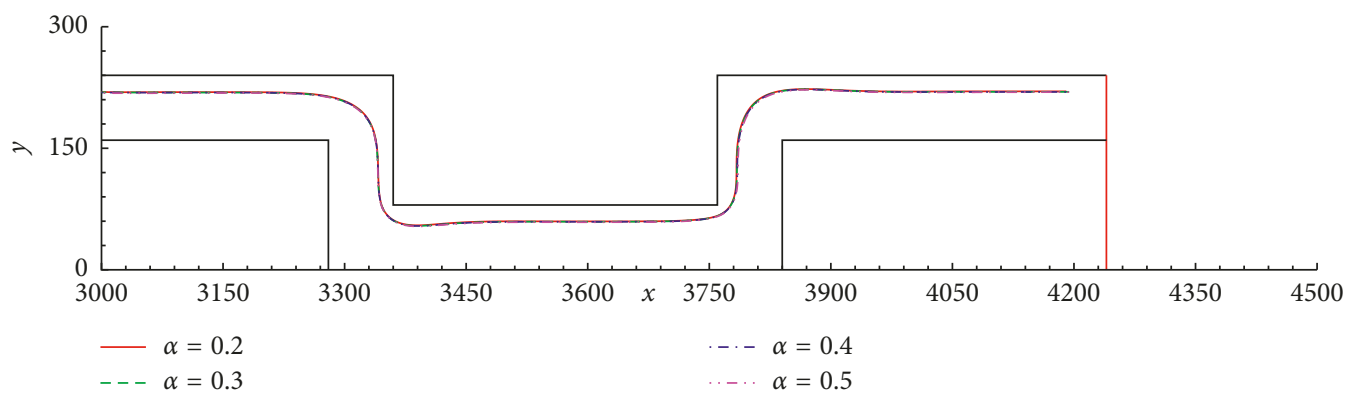

(a)

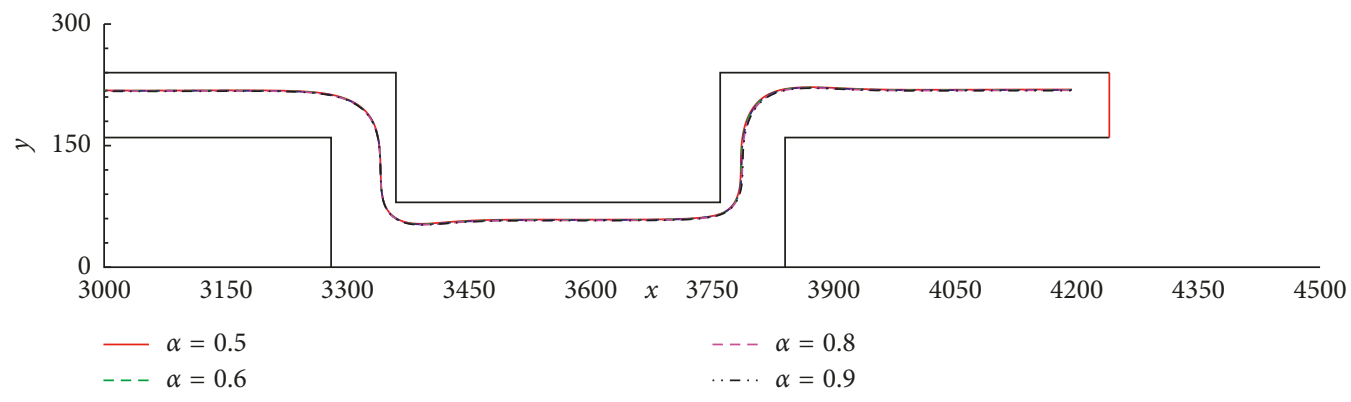

(b)

Figure 9: Particle trajectories for different density ratios when $\alpha<1$ : (a) $\alpha=0.2-0.5$ and (b) $\alpha=0.5-0.9$.

the effect of the particle density on the migration behavior of the particle in the serpentine channel in this section. The solid-to-fluid density ratio is defined as $\alpha=\rho_{\mathrm{p}} / \rho_{\mathrm{f}}$. All the results in the previous section treat the particle density $\rho_{\mathrm{p}}$ equal to the fluid density $\rho_{\mathrm{f}}$, that is, $\alpha=1$. To save the computational resources, some parameters are fixed at $\rho_{\mathrm{f}}=1, \operatorname{Re}=20$, and $q=0.3$ in this section.
We first consider the cases for which the fluid is heavier than the particle, that is, $\alpha<1$. Figure 9 shows the particle trajectories for different values of $\alpha$. It is interesting to find that no visible difference is observed for all the results shown, suggesting that the effect of the density ratio on the migration behavior of the particle in the serpentine channel is negligible for $\alpha<1$. However, things are very different for 


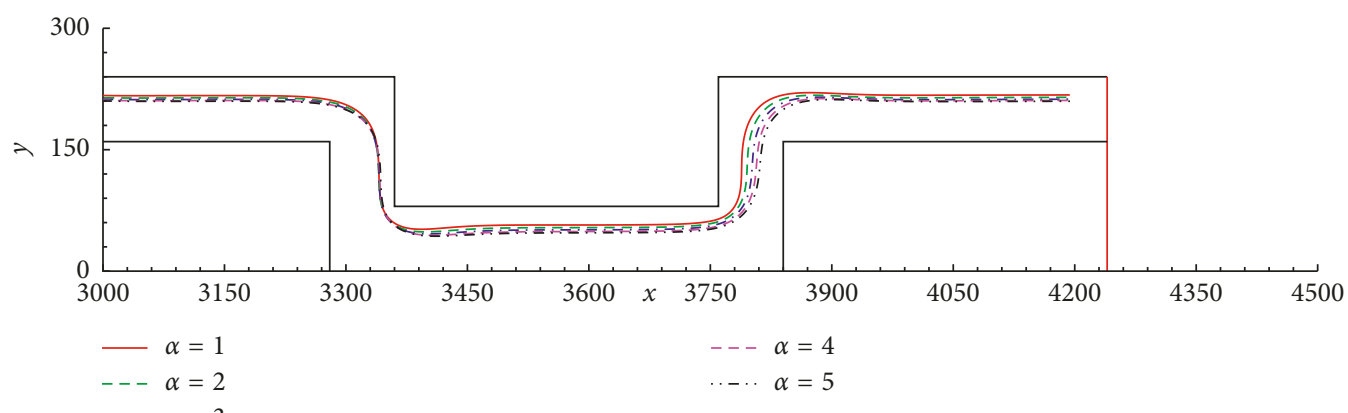

(a)

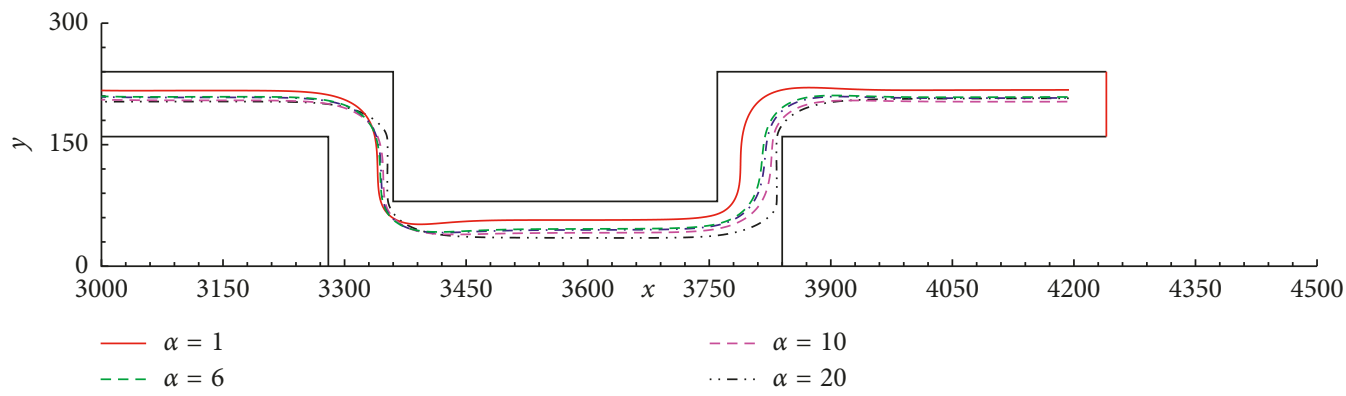

(b)

Figure 10: Particle trajectories for different density ratios when $\alpha>1$ : (a) $\alpha=1-5$ and (b) $\alpha=1-20$.

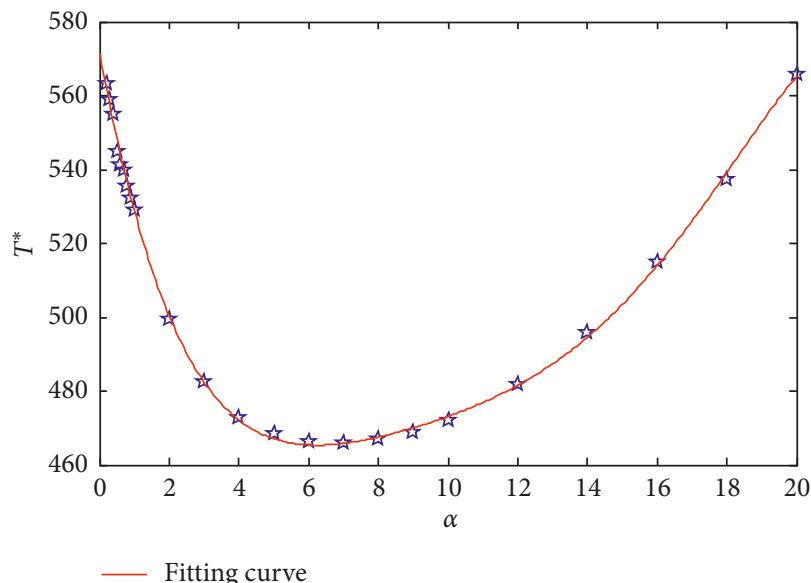

FIGURE 11: Time needed for the particle to travel in the channel for different values of $\alpha$.

$\alpha>1$, as shown in Figure 10. The particle is more likely to maintain its motion for larger density ratio, leading to the fact that the particle approaches closer to the channel wall after traveling in the straight section of channel. This trend is very significant when the particle is heavy such as $\alpha=10$ and 20, as shown in Figure 10(b).

Similarly, we also present the time needed for the particle to travel in the channel upon different values of $\alpha$ in Figure 11. The effect of density ratio on $T^{*}$ is significant. It is obvious that $T^{*}$ decreases as $\alpha$ increases initially and then increases afterwards. In addition, $T^{*}$ reaches its minimum value at $\alpha \approx 7$, as we can observe from Figure 11 . This suggests that it may take the shortest time for the particle to travel in the channel if it has a particular density. It is also interesting to find that for $\alpha<1$, the value of $T^{*}$ decreases as $\alpha$ increases despite that the particle trajectories (Figure 9) are almost identical, as shown in Figure 11. Finally, it is found that a quintic polynomial function like (8) fits the results quite well,

$$
\begin{aligned}
T^{*}= & -0.0006123 \alpha^{5}+0.03686 \alpha^{4}-0.8384 \alpha^{3} \\
& +9.486 \alpha^{2}-51.62 \alpha+571.7
\end{aligned}
$$

Finally, it should be stated that the present work only involves two-dimensional numerical simulation, which is different from the existing experimental work. For instance, the secondary flow may play an important role on the migration of particles as well as the interaction between particles in channel flows when the fluid inertia is significant, which cannot be taken into account in the present two-dimensional study. However, it is generally agreed that the two-dimensional simulations can be served as a tool for studying threedimensional flow characteristics when the Reynolds number is not large. As mentioned above, this work is the first step of investigation. We hope it casts a light on some features of particle migration in a serpentine channel. Certainly, the motion of spherical particles as well as their interactions in a threedimensional channel flow would be our future work.

\section{Conclusion}

In this work, the lattice Boltzmann method based on the momentum exchange scheme has been adopted to numerically 
study the migration of a particle in a serpentine channel. We focus on the effects of the Reynolds number (Re) as well as the initial position of the particle $(q)$ on the migration behavior of the particle in the channel. The Reynolds number ranges from 20 to 120 .

(1) The effect of Re on the final equilibrium position of the particle is significant, which is found to be more sensitive to the initial position of the particle when $\mathrm{Re}$ is small. For $\operatorname{Re}=120$, the largest Reynolds number studied in this work, the particle has a similar equilibrium position irrespective of its initial position for $q<0.5$ or $q>0.5$. Interestingly, results show that the particle almost stays on the channel centerline for the range of Re studied when $q=0.5$.

(2) It has been found that the particle is driven farther away from the channel wall after passing through each bend while increasing $\mathrm{Re}$, which is more significant for small values of $q$. This is due to the fact that the larger the Reynolds number, the larger the centrifugal force experienced by the particle.

(3) The solid-to-fluid density ratio $\alpha$ is central to the migration of the particle in the channel. It has been found that the time it takes for the particle to travel in the channel decreases as $\alpha$ increases initially and then increases afterwards. There exists a critical value of density ratio for which the particle travels fastest in the channel.

\section{Data Availability}

The data used to support the findings of this study are available from the corresponding author upon request.

\section{Conflicts of Interest}

The authors declare that they have no conflicts of interest.

\section{Acknowledgments}

This work was supported by the Zhejiang Provincial Natural Science Foundation of China (LY15A020004) and the National Key Research and Development Program of China (2017YFB0603700).

\section{References}

[1] C. D. Carlo, D. Irimia, R. G. Tompkins, and M. Toner, "Continuous inertial focusing, ordering, and separation of particles in microchannels," Proceedings of the National Academy of Sciences of the United States of America, vol. 104, no. 48, pp. 18892-18897, 2007.

[2] G. Segré and A. Silberberg, "Radial particle displacements in Poiseuille flow of suspensions," Nature, vol. 189, no. 4790, pp. 209-210, 1961.

[3] B. P. Ho and L. G. Leal, "Inertial migration of rigid spheres in two-dimensional unidirectional flows," Journal of Fluid Mechanics, vol. 65, no. 2, pp. 365-400, 1974.

[4] J. Feng, H. H. Hu, and D. D. Joseph, "Direct simulation of initial value problems for the motion of solid bodies in a Newtonian fluid. Part 2. Couette and Poiseuille flows," Journal of Fluid Mechanics, vol. 277, no. 1, pp. 271-301, 1994.

[5] T. W. Pan and R. Glowinski, "Direct simulation of the motion of neutrally buoyant circular cylinders in plane Poiseuille flow," Journal of Computational Physics, vol. 181, no. 1, pp. 260-279, 2002.

[6] B. Chun and A. J. C. Ladd, "Inertial migration of neutrally buoyant particles in a square duct: an investigation of multiple equilibrium positions," Physics of Fluids, vol. 18, no. 3, p. 031704, 2006.

[7] H. H. Yi, L. J. Fan, and Y. Y. Chen, "Lattice Boltzmann simulation of the motion of spherical particles in steady Poiseuille flow," International Journal of Modern Physics C, vol. 20, no. 6, pp. 831-846, 2009.

[8] S. D. Chen, T. W. Pan, and C. C. Chang, "The motion of a single and multiple neutrally buoyant elliptical cylinders in plane Poiseuille flow," Physics of Fluids, vol. 24, no. 10, p. 103302, 2012.

[9] M. Abbas, P. Magaud, Y. Gao, and S. Geoffroy, "Migration of finite sized particles in a laminar square channel flow from low to high Reynolds numbers," Physics of Fluids, vol. 26, no. 12, p. 123301, 2014.

[10] D. Jiang, W. Tang, N. Xiang, and Z. Ni, "Numerical simulation of particle focusing in a symmetrical serpentine microchannel," RSC Advances, vol. 6, no. 62, pp. 57647-57657, 2016.

[11] J. M. Martel and M. Toner, "Particle focusing in curved microfluidic channels," Scientific Reports, vol. 3, no. 1, p. 3340, 2013.

[12] L. L. Fan, Y. Han, X. K. He, L. Zhao, and J. Zhe, "Highthroughput, single-stream microparticle focusing using a microchannel with asymmetric sharp corners," Microfluidics and Nanofluidics, vol. 17, no. 4, pp. 639-646, 2014.

[13] S. S. Kuntaegowdanahalli, A. A. S. Bhagat, G. Kumar, and I. Papautsky, "Inertial microfluidics for continuous particle separation in spiral microchannels," Lab on a Chip, vol. 9, no. 20, pp. 2973-2980, 2009.

[14] T. Morijiri, M. Yamada, T. Hikida, and M. Seki, "Microfluidic counter flow centrifugal elutriation system for sedimentationbased cell separation," Microfluidics and Nanofluidics, vol. 14, no. 6, pp. 1049-1057, 2013.

[15] J. Zhang, S. Yan, R. Sluyter, W. Li, G. Alici, and N.-T. Nguyen, "Inertial particle separation by differential equilibrium positions in a symmetrical serpentine micro-channel," Scientific Reports, vol. 4, no. 1, p. 4527, 2014.

[16] Y. Liu and D. M. Nie, "Numerical simulation of particle motion in a curved channel," IOP Conference Series: Materials Science and Engineering, vol. 301, p. 012088, 2018.

[17] Y. H. Qian, D. D'Humieres, and P. Lallemand, "Lattice BGK model for Navier-Stokes equation," Europhysics Letters, vol. 17, no. 6, pp. 479-484, 1992.

[18] X. He and L. S. Luo, "Lattice Boltzmann model for the incompressible Navier-Stokes equation," Journal of Statistical Physics, vol. 88, no. 3-4, pp. 927-944, 1997. 


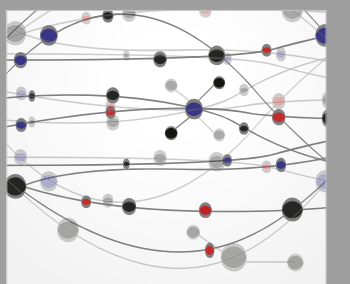

The Scientific World Journal
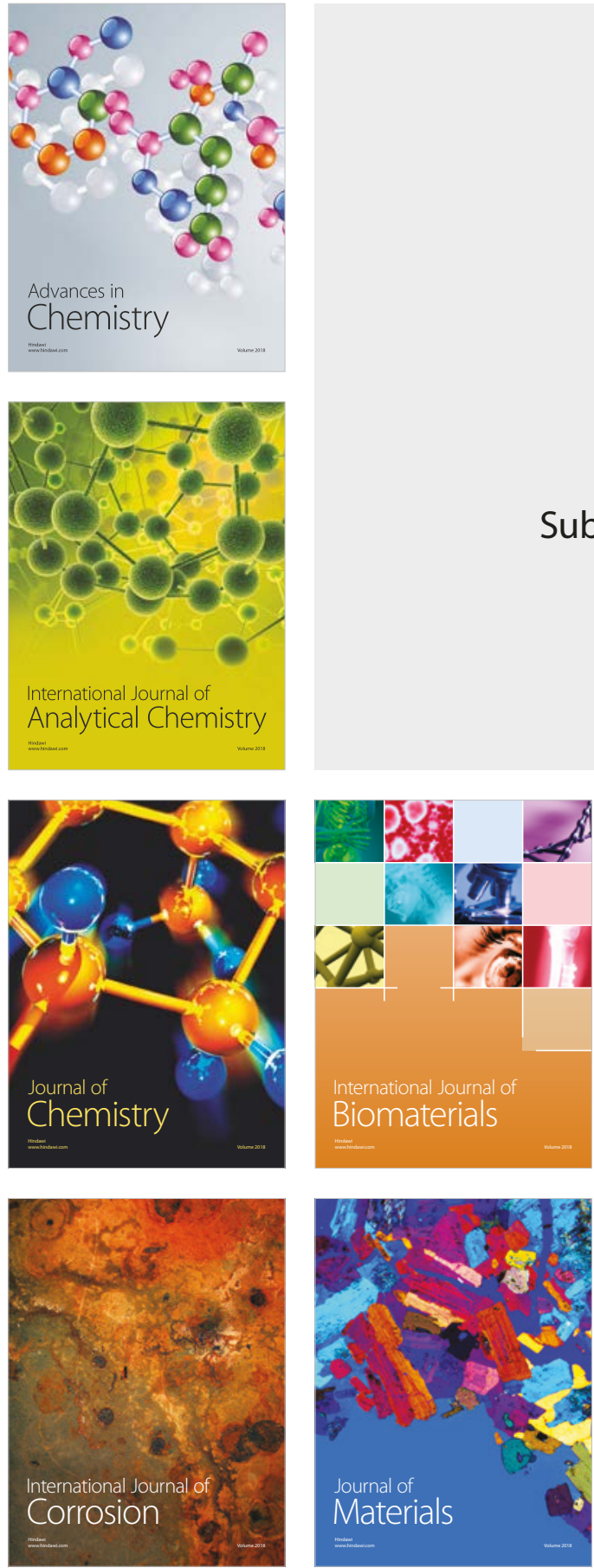

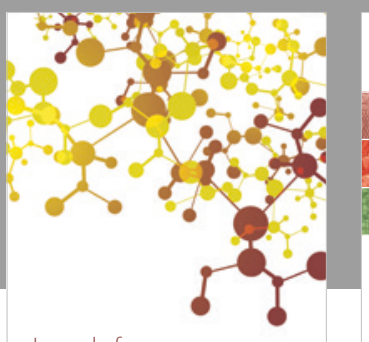

Journal of

Applied Chemistry
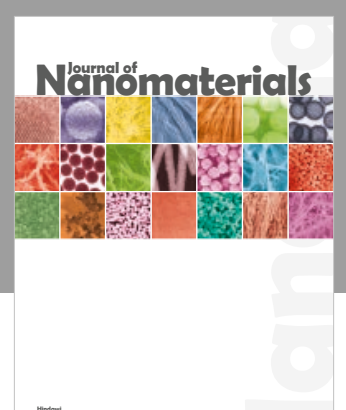

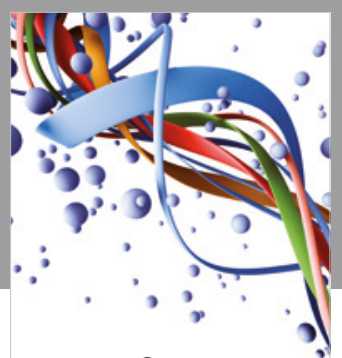

Scientifica

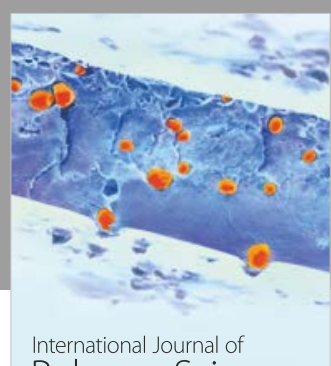

Polymer Science

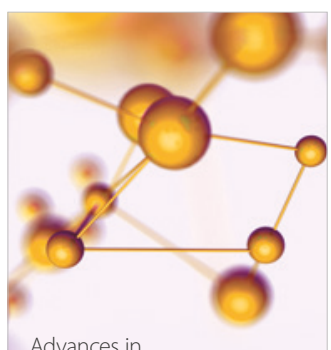

Physical Chemistry
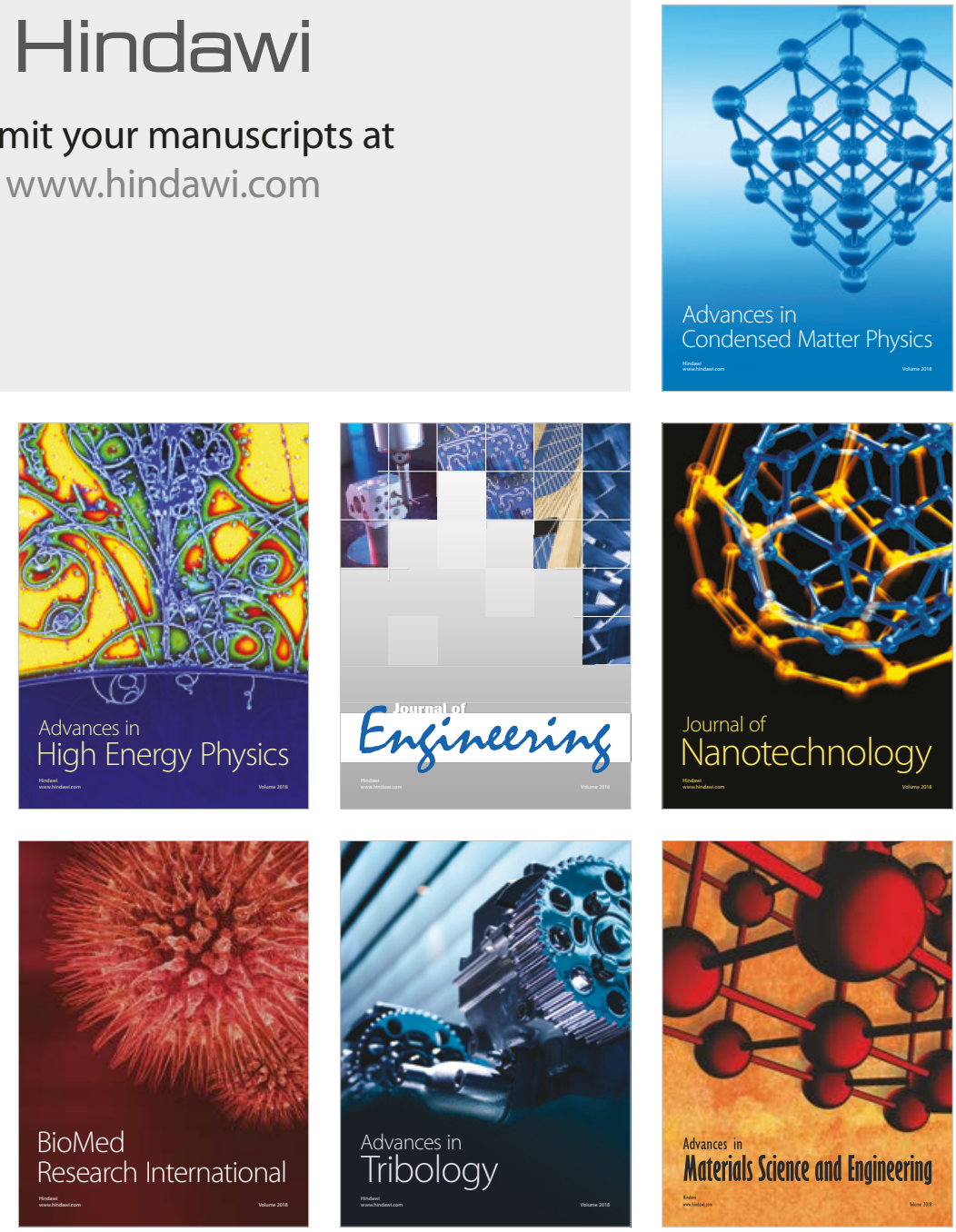